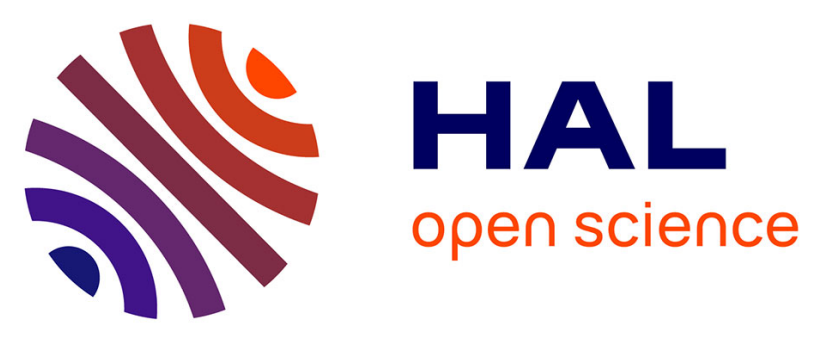

\title{
Size Effects in a Silica-Polystyrene Nanocomposite: Molecular Dynamics and Surface-enhanced Continuum Approaches
}

Denis Davydov, Evangelos Voyiatzis, Georges Chatzigeorgiou, Shengyuan Liu, Paul Steinmann, Michael C. Bohm, Florian Muller-Plathe

\section{To cite this version:}

Denis Davydov, Evangelos Voyiatzis, Georges Chatzigeorgiou, Shengyuan Liu, Paul Steinmann, et al.. Size Effects in a Silica-Polystyrene Nanocomposite: Molecular Dynamics and Surfaceenhanced Continuum Approaches. Soft Materials, 2014, 12 (Supplement 1), pp.S142-S151. 10.1080/1539445X.2014.959597 . hal-01201924

\section{HAL Id: hal-01201924 \\ https://hal.science/hal-01201924}

Submitted on 18 Sep 2015

HAL is a multi-disciplinary open access archive for the deposit and dissemination of scientific research documents, whether they are published or not. The documents may come from teaching and research institutions in France or abroad, or from public or private research centers.
L'archive ouverte pluridisciplinaire HAL, est destinée au dépôt et à la diffusion de documents scientifiques de niveau recherche, publiés ou non, émanant des établissements d'enseignement et de recherche français ou étrangers, des laboratoires publics ou privés. 


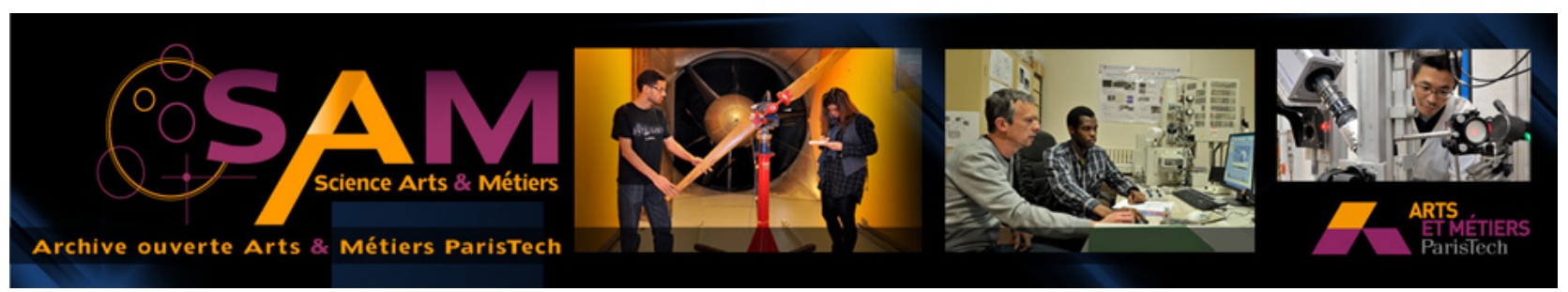

\section{Science Arts \& Métiers (SAM)}

is an open access repository that collects the work of Arts et Métiers ParisTech researchers and makes it freely available over the web where possible.

This is an author-deposited version published in: http://sam.ensam.eu

Handle ID: .http://hdl.handle.net/10985/10051

\section{To cite this version :}

Denis DAVYDOV, Evangelos VOYIATZIS, George CHATZIGEORGIOU, Shengyuan LIU, Paul STEINMANN, Michael C. BOHM, Florian MULLER-PLATHE - Size Effects in a Silica-Polystyrene Nanocomposite: Molecular Dynamics and Surface-enhanced Continuum Approaches - Soft Materials - Vol. 12, nSupplement 1, p.S142-S151 - 2014 


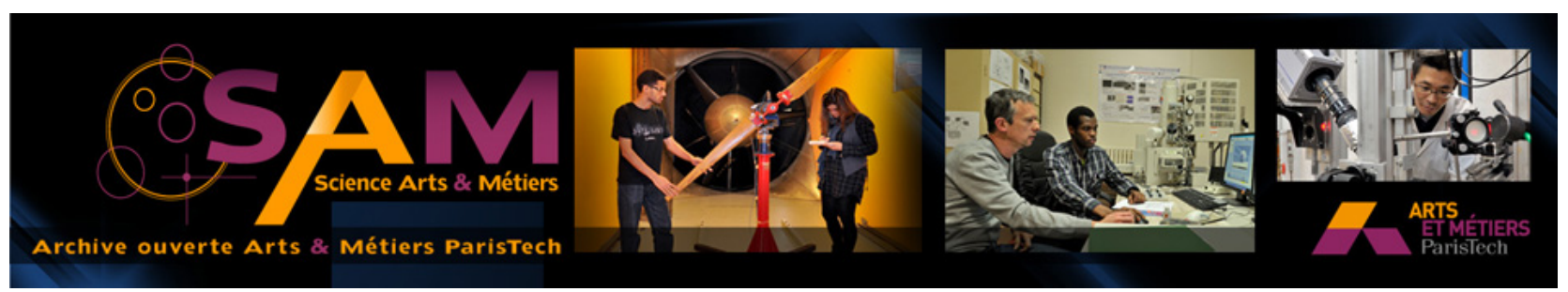

\section{Science Arts \& Métiers (SAM)}

is an open access repository that collects the work of Arts et Métiers ParisTech researchers and makes it freely available over the web where possible.

This is an author-deposited version published in: http://sam.ensam.eu

Handle ID: .http://hdl.handle.net/null

\section{To cite this version :}

Denis DAVYDOV, Evangelos VOYIATZIS, George CHATZIGEORGIOU, Shengyuan LIU, Paul STEINMANN, Michael C. BOHM, Florian MULLER-PLATHE - Size Effects in a Silica-Polystyrene Nanocomposite: Molecular Dynamics and Surface-enhanced Continuum Approaches - Soft Materials - Vol. 12, p.S142-S151 - 2014 


\title{
Size Effects in a Silica-Polystyrene Nanocomposite: Molecular Dynamics and Surface-enhanced Continuum Approaches
}

\author{
DENIS DAVYDOV ${ }^{1 *}$, EVANGELOS VOYIATZIS ${ }^{2}$, GEORGE CHATZIGEORGIOU ${ }^{3}$, SHENGYUAN LIU ${ }^{2}$, PAUL STEINMANN ${ }^{1}$, \\ MICHAEL C. BÖHM², and FLORIAN MÜLLER-PLATHE ${ }^{2}$ \\ ${ }^{1}$ Chair of Applied Mechanics, University of Erlangen-Nuremberg, Erlangen, Germany \\ ${ }^{2}$ Eduard-Zintl-Institut für Anorganische und Physikalische Chemie and Center of Smart Interfaces, Technische Universität Darmstadt, \\ Darmstadt, Germany \\ ${ }^{3}$ LEM3-UMR 7239 CNRS, Arts et Métiers ParisTech Metz-Lorraine, Metz, France
}

\begin{abstract}
Size effects in a system composed of a polymer matrix with a single silica nanoparticle are studied using molecular dynamics and surface-enhanced continuum approaches. The dependence of the composite's mechanical properties on the nanoparticle's radius was examined. Mean values of the elastic moduli obtained using molecular dynamics were found to be lower than those of the polystyrene matrix alone. The surface-enhanced continuum theory produced a satisfactory fit of macroscopic stresses developing during relaxation due to the interface tension and uniaxial deformation. Neither analytical nor finite-element solutions correlated well with the size-effect in elastic moduli predicted by the molecular dynamics simulations.
\end{abstract}

\section{Introduction}

Molecular dynamics (MD) simulations have been used extensively to analyse size dependent properties of nanocomposite (NC) systems (1-8). Such NCs are widely used in industry due to their attractive mechanical properties. Their behavior, however, cannot be predicted from mechanical properties of its components alone. Reliable quantification is only possible if the significant role of the interphase is taken into account.

Several experimental studies have demonstrated an increase in NC Young's modulus with decreasing nanoparticle (NP) size $(9,10)$. The same trend has been observed in MD simulations $(6$, $7,11)$. On the other hand, the non-monotonic behavior of elastic modulus with respect to the mass-fraction of the NP filler was also reported (12). In some cases, the NC's elastic moduli are even lower than those of the matrix $(13,14)$. From the experimental perspective, NCs could have inferior mechanical properties due to the poor dispersion (15).

Fully resolved atomistic or even coarse-grained (CG) MD simulations of microscale systems are still computationally expensive. To overcome this limitation several continuum mechanics (CM) approaches have been developed. One possibility is to introduce an effective interphase $(11,16,17)$, which is based on the observation that the polymer particles in the vicinity of the NP exhibit properties that are distinct from those of the bulk polymer $(1,2,8,13,14,18)$. The width of the interphase depends on the chosen polymer matrix but may be insensitive to the NP size (18). For this reason a fixed value is typically assumed in micromechanical models (17). Given a constant NP volume fraction, this implies that the volume fraction of the interphase increases as the size of the NP decreases. This in turn introduces size-dependence to the solution. Isotropic elastic moduli of the interphase are usually identified by comparing MD and CM predictions $(14,17)$. An alternative approach that introduces sizeeffects dates back to works of Gurtin and Murdoch (19) where surface elasticity theory was introduced. At the boundary of the domain this theory introduces an independent surface free energy that depends on the curvature of the manifold, surface deformation gradient and surface stress. This approach allows different effects related to surface energy in solids and liquids to be captured [see (20) and references therein]. This will be referred to as surface-enhanced continuum (SEC) theory. Its application to NC systems is the focus of the present contribution. The validation of the SEC theory as compared to atomistic simulations has been performed by the authors for a copper plate with a nano hole (21, 22). The basis for such validation is a theoretical link between the atomistic and the continuum formulation (23-25) that provides expressions for local continuum fields (velocity, Cauchy stress, energy) in terms of atomistic quantities. There are also 
analytical approaches to predict effective moduli within the SEC theory (26-28).

In this contribution we discuss size dependent mechanical properties of a NC composed of a bare silica NP embedded into an atactic polystyrene (PS) matrix. Local structural and dynamical properties of such systems have been studied extensively both in atomistic and coarse-grained resolution $(1,2)$. While there has been a number of studies on the mechanical properties of NCs (6-8), to our best knowledge no work has been conducted using the MD model employed here for the purpose of studying the size-effect in mechanical properties. Moreover, often it is only Young's modulus $(3,8)$ or elastic constants from unidirectional and hydrostatic loading (7) that are reported for NC systems. Marcadon et al. (17) performed three tensile tests and three shear tests for polymer nanocomposites; however only the eqvivalent isotropic moduli were presented. In a previous study (3), the Young's modulus of a silica-PS composite has been reported for different filling densities. The size effect, however, has not been studied. In the present work, we determine the complete set of elastic moduli for NCs.

This contribution serves two purposes. First, we utilize MD simulations to observe the macroscopic size effects for the considered NC systems. Several NCs with different NP radii are considered in order to observe size dependent mechanical properties. Second, we apply SEC theory to describe the observed size effect. To the best of our knowledge this has not been previously documented. In this work, it is hypothesized that the NC's volume-fraction needs to be interpreted differently in the context of MD and SEC theories. SEC is proven to be a promising approach to consider nanoscale problems (20-22, 29-31). For both the MD and CM simulations we avoid any constraints, such as making NPs rigid (8).

The paper is organized as follows: The Molecular Dynamics Methods section provides all details related to the MD simulations. In the Surface-Enhanced Continuum Theory section we briefly remind the reader of the fundamentals of SEC theory. The Mean Field Homogenization section provides a brief introduction to analytical homogenization methods. In the Initial Configurations in MD and SEC section, we discuss core differences between SEC and MD initial configurations for $\mathrm{NC}$ systems. A method to consistently relate the two approaches, as well as to study size-effects, is proposed. In the final sections, results are discussed and conclusions are presented.

\section{Molecular Dynamics Methods}

The nanocomposites considered in this work consist of a single bare silica nanoparticle dispersed in an atactic polystyrene matrix. Each polymeric chain has a length of 200 monomers with a molecular weight of $20845 \mathrm{amu}$. The systems are studied in the glass state, i.e., below their glass transition temperature, $T_{g}$. Furthermore, we consider two additional systems from which we extract the bulk mechanical properties of the individual phases. The first system consists of 300 polystyrene chains where each chain is 200 monomers long, i.e., they have the same length as the polymer matrix of the NCs. The second system is a trigonal $\alpha$-quártz silica crystal composed of $10125 \mathrm{SiO}_{2}$ units.
The nanocomposites are represented in a coarse-grained (CG) formulation. Two different types of $\mathrm{CG}$ beads are employed in the description of the $\mathrm{SiO}_{2}$ units of the nanoparticle which differ in their coordination number (1). We have so-called core beads with full coordination as well as surface beads. Similar to previous studies $(1,3,32)$, each styrene monomer is described by a CG bead where the interaction center is located at the center-of-mass of the monomer. Two different beads ( $\mathrm{R}$ and $\mathrm{S}$ ) are required to maintain the information on the chirality of a PS chain. The employed CG force field has been obtained by Iterative Boltzmann Inversion (33) at $590 \mathrm{~K}$ and $101.3 \mathrm{kPa}$; more details are given in Ghanbari et al. (1).

Initial configurations of all systems were prepared at the melt state with a temperature and pressure of $590 \mathrm{~K}$ and $101.3 \mathrm{kPa}$, respectively. The silica nanoparticle is cut from an $\alpha$-quartz crystal structure by defining a sphere with radius $\mathrm{R}^{*}$ and removing all atoms outside this sphere. The NP, with a mass of $\mathrm{M}_{\mathrm{np}}$, is inserted into an empty simulation box. The surrounding polymer chains, with a total mass of $\mathrm{M}_{\mathrm{p}}$, are distributed by a self-avoiding random walk method (1). The systems are equilibrated in the isothermal-isobaric (N,P,T) statistical ensemble for $20 \mathrm{~ns}$. The Nosé-Hoover thermostat and barostat are employed to control the temperature and the pressure of the system (34) with coupling times of $0.2 \mathrm{ps}$ and $5.0 \mathrm{ps}$. The equations of motion are integrated using the velocity-Verlet scheme (35) with a time step of $0.005 \mathrm{ps}$. A $1.5 \mathrm{~nm}$ cutoff for the summation of the non-bonded interactions is employed. All MD simulations were performed using the LAMMPS code (36).

The nanocomposites are cooled down below their $T_{g}$ in order to obtain amorphous glass structures. The $T_{g}$ of pure PS in CG resolution has been reported to be $170 \mathrm{~K}(3)$. The temperature is decreased with a constant cooling rate of $9.8 \mathrm{~K} / \mathrm{ns}$ for $50 \mathrm{~ns}$, which is in line with the one employed in a previous study (3). The systems are allowed to further relax for $5 \mathrm{~ns}$ in the isothermal-isobaric $(\mathrm{N}, \mathrm{P}, \mathrm{T})$ ensemble once the final temperature of $100 \mathrm{~K}$ is reached. The NP radius at the final temperature, $\mathrm{R}_{\mathrm{MD}}$, see Fig. 1(a), differs in general from the initial radius that has been used to create the nanoparticle, $R^{*}$. The length of the simulation box of a system at the final temperature is denoted by $\mathrm{L}_{\mathrm{MD}}$.

The mechanical properties and the elastic moduli of the nanocomposites are computed by applying small-strain deformations to the amorphous glass structures without making the assumption that the materials are isotropic (37). Four different deformation modes, namely uniaxial tension, uniaxial deformation, volumetric deformation, and simple shear, are employed; they are presented schematically in Fig. 2. The reported elastic moduli are averaged for both positive and negative strains. A deformation rate of $10 \mathrm{~nm} / \mathrm{ns}$ was applied in all cases until a $1 \%$ deformation was achieved. After the box was deformed, the system was allowed to relax for $10 \mathrm{~ns}^{1}$ in the canonical $(\mathrm{N}, \mathrm{V}, \mathrm{T})$ ensemble. For uniaxial tension the NoséHoover barostat was considered in transversal directions. The

${ }^{1}$ In [5] the authors concluded that NCs might have viscoelastic behavior. Thus, the averaged stresses and the resulting elastic moduli could also depend on the chosen relaxation time. 


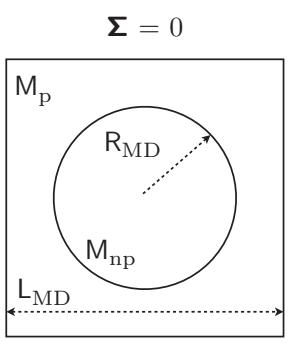

(a) fully relaxed

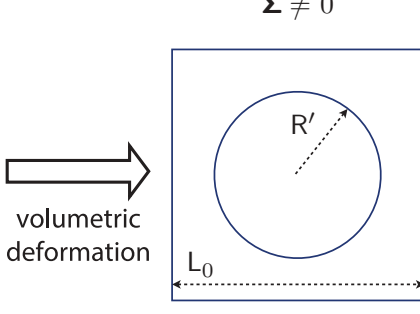

(b) confined

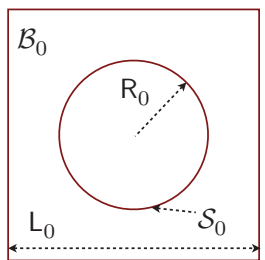

(c) material/reference

Fig. 1. (a) Initial configuration of the system for the MD simulations; (b) The continuum solution after relaxation required due to the introduction of the interface energy; (c) Initial configuration used in the SEC theory. In the absence of the interface energy, the CM system would have been in a stress free state. $\Sigma$ denotes the macroscopic Cauchy stress, $\mathrm{M}_{n p}$ and $\mathrm{M}_{\mathrm{p}}$ are masses of NP and PS, respectively; $\mathrm{L}_{\mathrm{MD}}$ denotes the representative volume element (RVE) size in the fully relaxed state, whereas $\mathrm{L}_{0}$ and $\mathrm{R}_{0}$ denote the RVE characteristic length and NP radius in the material configuration of the SEC theory. $\mathrm{R}^{\prime}$ indicates the NP radius after relaxation due to interface tension. Note, $\mathrm{L}_{0} \neq \mathrm{L}_{\mathrm{MD}}$ and $\mathrm{R}^{\prime} \neq \mathrm{R}_{0}, \mathrm{R}_{0} \neq \mathrm{R}_{\mathrm{MD}}, \mathrm{R}^{\prime} \neq \mathrm{R}_{\mathrm{MD}}$

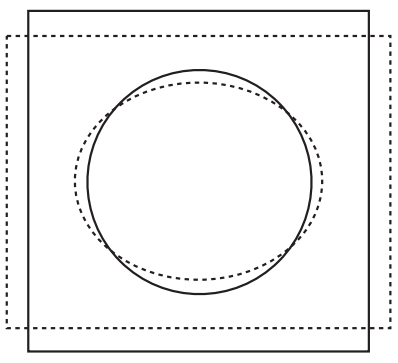

(a) uniaxial tension

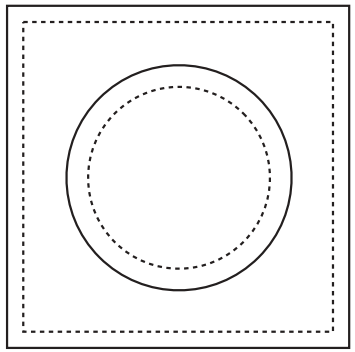

(c) volumetric deformation

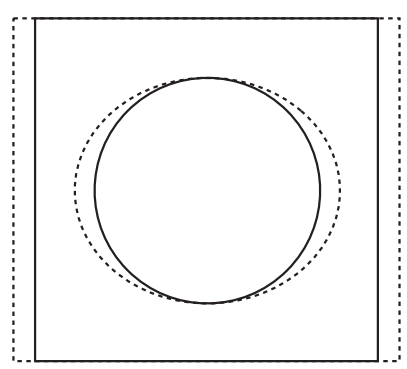

(b) uniaxial deformation

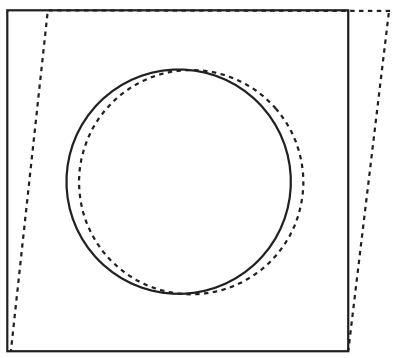

(d) simple shear
Fig. 2. Illustration of different deformation modes considered in this study. Solid and dashed lines denote initial and deformed configurations, respectively.

Cauchy stress tensor $\boldsymbol{\Sigma}$ is computed on the basis of the atomic Virial pressure (34), which is recorded for the last $5 \mathrm{~ns}$ of the relaxation period and is sampled every $25 \mathrm{ps}$. The elastic properties of the $\alpha$-quartz silica are obtained at $0 \mathrm{~K}$. They are computed as the first derivatives of the macroscopic stress with respect to shear and uniaxial deformation gradients of $1 \%$.

For those moduli that linearly depend on random variables, the standard deviation can be calculated. ${ }^{2}$ Note the sum of two independent random variables with normal distribution $X \sim$

${ }^{2}$ For nonlinear cases, the resulting standard deviation of the normal distribution could be estimated by sampling random variables. However, in order to keep on figures easy to digest, we did not indicate results from this approach.
$N\left(\mu_{x}, \sigma_{x}^{2}\right)$ and $X \sim N\left(\mu_{y}, \sigma_{y}^{2}\right)$ has normal distribution $X+$ $Y \sim N\left(\mu_{x}+\mu_{y}, \sigma_{x}^{2}+\sigma_{y}^{2}\right)$.

General anisotropic elastic moduli obtained from uniaxial deformation and simple shear (in Voight notation) are denoted by $\mathrm{C}^{\mathrm{ij}}, \mathrm{i}, \mathrm{j}=\overline{1,6}$; the resulting $\mathrm{C}^{\mathrm{ij}}$ are symmetrised. Thus, the standard deviation related to off-diagonal components is expected be higher. Young's moduli obtained from uniaxial tension in each direction will be denoted by $\mathrm{E}^{\mathrm{i}}, \mathrm{i}=\overline{1,3}$; finally the bulk modulus is denoted by K. Recall that under the assumption of isotropy any pair of elastic moduli determine all the others. For example, when expressed in terms of Lamé moduli $\lambda$ and $\mu$, the elastic moduli read: $\mathrm{C}^{12}=\mathrm{C}^{13}=\mathrm{C}^{23}=\lambda, \mathrm{C}^{44}=\mathrm{C}^{55}=\mathrm{C}^{66}=\mu$, and $\mathrm{C}^{11}=\mathrm{C}^{22}=\mathrm{C}^{33}=\lambda+2 \mu$, and the others are zero. The isotropic elastic moduli are obtained by a least square fit of (generally anisotropic) elastic moduli resulting from uniaxial deformation and shear.

\section{Surface-Enhanced Continuum Theory}

This section provides a brief introduction to the SEC theory. Theoretical and numerical details can be found elsewhere (38, $39)$. The notation adopted in the present study is explained in Appendix 1.

Consider a continuum body that takes material (reference) configuration $\mathcal{B}_{0}$ at time $t_{0}$, as illustrated in Fig. 1(c). For the $\mathrm{NC}$ systems, the material configuration is defined by the representative volume element (RVE) size $L_{0}$ and the NP radius $R_{0}$. At this reference configuration, both NP and PS are considered to be homogeneous in space with density equal to the bulk phase: $\rho_{p}$ and $\rho_{n p}$, respectively. Consequently, the volume occupied by each phase is provided by $\mathrm{V}_{\mathrm{p}}=\mathrm{M}_{\mathrm{p}} / \rho_{p}$ and $\mathrm{V}_{\mathrm{np}}=\mathrm{M}_{\mathrm{np}} / \rho_{n p}$, respectively. Thus, mass fraction is constant if and only if the volume fraction of phases, or equivalently $\mathrm{R}_{0} / \mathrm{L}_{0}$, is constant.

The body is mapped to the spatial (current) configuration $\mathcal{B}_{t}$ at time $t>t_{0}$ via volume deformation map $\varphi=\varphi(X)$. Material points in the material and spatial configurations are denoted by $\boldsymbol{X}$ and $\boldsymbol{x}$, respectively. The (bulk) deformation gradient is denoted by $\boldsymbol{F}$, and maps material line elements of $\mathrm{d} \boldsymbol{X} \in \mathcal{B}_{0}$ to spatial line elements $\mathrm{d} \boldsymbol{X} \in \mathcal{B}_{t}$ via $\mathrm{d} \boldsymbol{X}=\boldsymbol{F} \cdot \mathrm{d} \boldsymbol{X}$. 
Let $\mathcal{S}_{0}$ and $\mathcal{S}_{t}$ denote a zero thickness interface in the material and spatial configurations respectively. The outward unit normal in the material configurations is denoted by $\boldsymbol{N}$. Material points at the interface are denoted $\widehat{\boldsymbol{X}}$ and $\widehat{\boldsymbol{x}}$ in the material and spatial configurations and are attached to the volume, i.e., $\widehat{\boldsymbol{X}}=\left.\boldsymbol{X}\right|_{\mathcal{S}_{0}}$. Furthermore, it is assumed that the interface is of a material type, i.e., it remains attached to the body and thus $\widehat{\boldsymbol{x}}=\left.\boldsymbol{x}\right|_{\mathcal{S}_{t}}$.

In ordinary first gradient continuum theory, the bulk free energy is postulated to be a function of the (bulk) deformation gradient $\Psi=\Psi(\boldsymbol{F})$. In the current study, a hyperelastic neoHookean material model is employed. It is parametrised by the Lamé moduli ${ }^{3} \lambda$ and $\mu$ as $\Psi(\boldsymbol{F})=1 / 2 \lambda \ln ^{2} J+1 / 2 \mu[\boldsymbol{F}: \boldsymbol{F}-$ $3-2 \ln J$ ], where $J=\operatorname{det} \boldsymbol{F}$ denotes the (bulk) Jacobian. The corresponding values for each phase in the $\mathrm{NC}$ will be discussed later.

In the SEC theory the surface free energy $\hat{\Psi}=\hat{\Psi}(\widehat{\boldsymbol{F}})$ is introduced, where $\widehat{\boldsymbol{F}}$ denotes the surface deformation gradient. In the absence of body forces, the quasistatic balances of linear momentum are given by:

$$
\begin{array}{ll}
\operatorname{Div} \boldsymbol{P}=\boldsymbol{0} & \text { in } \mathcal{B}_{0}, \\
\widehat{\operatorname{Div}} \hat{\boldsymbol{P}}=\boldsymbol{P} \cdot \boldsymbol{N} & \text { on } \mathcal{S}_{0},
\end{array}
$$

where $\boldsymbol{P}=\partial \Psi / \partial \boldsymbol{F}$ and $\widehat{\boldsymbol{P}}=\partial \widehat{\Psi} / \partial \widehat{\boldsymbol{F}}$ denote the bulk and surface Piola stress, respectively.

The surface free energy is considered to be of neo-Hookean hyperelastic type: $\widehat{\Psi}(\widehat{\boldsymbol{F}})=1 / 2 \widehat{\lambda} \ln ^{2} \widehat{J}+$ $1 / 2 \widehat{\mu}[\widehat{\boldsymbol{F}}: \widehat{\boldsymbol{F}}-2-2 \ln \widehat{J}]+\widehat{\gamma} \widehat{J}$, where $\widehat{J}$ denotes the surface Jacobian. The parameter $\widehat{\gamma}$ accounts for interface tension.

To solve CM problem, the following strategy was employed: i) rewrite Eq. (1) and Eq. (2) in the weak form; ii) introduce a finite element basis for bulk and interface displacements; iii) linearize and apply the Newton-Raphson method; and iv) solve the resulting sparse system of linear algebraic equations. The continuum formulation is implemented using the open-source finite element (FE) method library deal.II (40). The current implementation is an extension of the surface-elasticity code, developed by McBride et al. (38), to interface problems.

If the interface tension $\widehat{\gamma}$ is not zero, even in the absence of macroscopic loading, the material configuration is not in equilibrium. In other words, the reference state does not satisfy Eq. (1) and Eq. (2). The equilibrium configuration is found by solving Eq. (1) and Eq. (2) under zero boundary conditions. If the size of the RVE is fixed, for isotropic problems, only the NP radius will change, but not its shape (Fig. 1b). It is important to note that in this configuration the macroscopic stresses are not zero. We call this initial solution step 'relaxation under interface tension'.

Also note that the surface parameters introduce a length scale into the boundary value problem; namely, the ratio between surface elastic parameters and bulk has a dimension of length. Therefore, the solution will be size dependent. This size effect will be studied by considering different material configurations where $\mathrm{R}_{0} / \mathrm{L}_{0}$ is constant. This implies a constant mass fraction of the two phases as discussed earlier. In particular, we will focus

${ }^{3}$ For isotropic materials the Hooke's law reads $\sigma=2 \mu \boldsymbol{\epsilon}+\lambda \operatorname{tr}(\boldsymbol{\epsilon}) \boldsymbol{I}$, where $\sigma$ is stress, $\boldsymbol{\epsilon}$ is the strain tensor and $\boldsymbol{I}$ is the second order identity tensor. on stresses developing due to relaxation under interface tension as well as macroscopic elastic moduli of the NCs. The latter are obtained by applying an uniaxial deformation gradient and assuming isotropic macroscopic behavior.

\section{Mean Field Homogenization}

In addition to the FE implementation of the SEC theory, analytical homogenization strategies are also considered in this work (Fig. 3). One of the widely used approaches to obtain properties of a matrix-inclusion composite is the Mori-Tanaka (MT) meanfield theory (41), which is based on Eshelby's solution of an inhomogeneity in an infinite medium (42). Note that this approach does not take into account properties of an interface/interphase, but rather it indicates the limiting values at a length scale where the size-effect is not pronounced.

Analytical homogenization schemes have been recently extended to a SEC scenario (28) (Fig. 3a), however the surface/interface tension is not considered. Results obtained by this analytical method will be denoted by $\mathrm{SEC}_{\mathrm{AN}}$.

Finally, it is interesting to apply micro-mechanics meanfield theories that explicitly consider interphases in the NC (see Fig. 3b). The relevance of their application is based on the observation that the polymer particles in the vicinity of the NP exhibit properties that differ from those of the bulk polymer $(1,2,8$, $13,14,18)$. Therefore, such an interphase is considered to have distinct elastic properties. For the current study we utilize the approach proposed in Marcadon et al. (17) and denote its results by MM. Based on a previous study (1), the interphase thickness of NC systems is assumed to be $2.0 \mathrm{~nm}$, whereas the NP radius is assumed to be equal to $\mathrm{R}_{0}$.

In all analytical solutions, isotropic small strain elastic behavior is assumed.

\section{Initial Configurations in MD and SEC}

As it is clear from the two preceding sections, MD and SEC consider principally different initial configurations (Fig. 1a and 1c). In particular, SEC starts from the NC configuration consistent with the macroscopic case, i.e., the total mass of PS and NP and their volumes are related by bulk densities. In contrast, in the

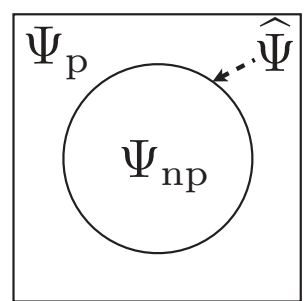

(a) interface

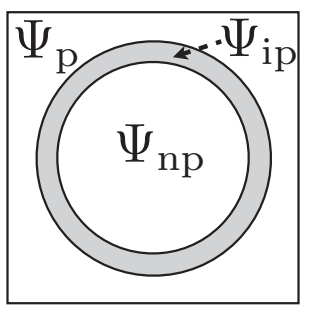

(b) interphase
Fig. 3. RVEs as considered in different analytical homogenization approaches. In interphase approaches, three phases with different mechanical properties are considered: NP, bulk PS, and interphase. In contrast the interface description introduces a free energy associated with the interface. By $\Psi$ we denote the free energy associated with the matrix $\Psi_{\mathrm{p}}$, the inclusion $\Psi_{\mathrm{np}}$, the interface $\hat{\Psi}$, or the interphase $\Psi_{\text {ip }}$. 
Table 1. A summary of NC systems considered in this study to observe the size-effect.

\begin{tabular}{cccccccc}
\hline & \# $\mathrm{SiO}_{2}$ beads & $\mathrm{R}^{*}[\mathrm{~nm}]$ & \# PS chains & $\mathrm{R}_{0}[\mathrm{~nm}]$ & $\mathrm{L}_{0}[\mathrm{~nm}]$ & $\mathrm{L}_{\mathrm{MD}}[\mathrm{nm}]$ & $\frac{\mathrm{M}_{\mathrm{np}}}{\mathrm{M}_{\mathrm{np}}+\mathrm{M}_{\mathrm{p}}}$ \\
\hline 1 & 1174 & 2.2 & 75 & 2.359 & 13.190 & 13.364 & 0.04584 \\
2 & 1719 & 2.5 & 110 & 2.679 & 14.991 & 15.153 & 0.04577 \\
3 & 2312 & 2.75 & 148 & 2.957 & 16.549 & 16.745 & 0.04575 \\
4 & 3000 & 3.0 & 192 & 3.225 & 18.050 & 18.227 & 0.04576 \\
5 & 4372 & 3.4 & 280 & 3.656 & 20.469 & 20.594 & 0.02390 \\
6 & 7108 & 4.0 & 455 & 4.299 & 24.065 & 24.240 & 0.04573 \\
\hline
\end{tabular}

$\mathrm{R}^{*}$ denotes the trimming radius and $\mathrm{L}_{\mathrm{MD}}$ denotes the equilibrium length of the simulation domain. $\mathrm{M}_{\mathrm{p}}$ and $\mathrm{M}_{\mathrm{np}}$ denote the total mass of PS and NP, respectively. Finally, $\mathrm{L}_{0}$ and $\mathrm{R}_{0}$ define the $\mathrm{NC}$ in the reference $\mathrm{CM}$ configuration, where its density is considered to be homogeneous.

MD approach the initial configuration is fully equilibrated and the densities of each phase are not homogeneously distributed in space.

Nonetheless, the same mass of each phase should be considered in both MD and SEC. Thus, knowing $\mathrm{M}_{\mathrm{p}}$ and $\mathrm{M}_{\mathrm{np}}$ and bulk densities $\rho_{\text {np }}$ and $\rho_{\mathrm{p}}$, from trivial geometrical considerations, one could obtain the corresponding values for $L_{0}$ and $R_{0}$ that define the domain in the material configuration of the SEC theory (Fig. 1c). This approach is similar to a Gibbs dividing surface construction (43), which was used by Shenoy (44) to determine the thickness of the crystalline slab.

Within the MD framework, the NC size-effect is studied by producing different configurations with $\mathrm{R}_{0} / \mathrm{L}_{0} \approx$ const (Table 1). This implies that the number of chains has to be adjusted to obtain configurations with close-to-constant mass fraction $\mathrm{M}_{\mathrm{p}} / \mathrm{M}_{\mathrm{np}} \approx$ const. As the length of PS chains is kept fixed, the total mass of PS varies discretely and thus it is generally possible to keep the mass-fraction only approximately constant. However, as it is evident from Table 1, variations are low.

Following our previous SEC work (21), a single configuration with a constant mass fraction is chosen (row 4 in Table 1 ). As the length scale is introduced to the problem by surface parameters, the size effect is studied by solving the CM equations on the same mesh while appropriately scaling the surface elastic parameters.

In order to arrive at comparable configurations for both approaches, one needs to deform volumetrically the relaxed MD configuration (Fig. 1a) to attain the same volume as in the material continuum configuration (Fig. 1b). This is achieved using the Nosé-Hoover thermostat coupled with the change of the RVE in 2.5 ns. Comparing $\mathrm{L}_{\mathrm{MD}}$ and $\mathrm{L}_{0}$ in Table 1 , we conclude that the required volumetric deformation is compressive. At this point both $\mathrm{CM}$ and $\mathrm{MD}$ configurations are expected to be fully equivalent with comparable macroscopic quantities such as the Cauchy stress and elastic moduli.

Note that one could allow the RVE size to relax in the CM formulation and thus obtain a stress-free configuration. However, such an approach is inconvenient from the implementation point of view because the FE approach is displacement driven. In particular, given the specified boundary conditions Eq. (1) and Eq. (2) are solved. The resulting macroscopic stress is obtained as an integral over $\mathcal{B}_{0}$ and $\mathcal{S}_{0}$.

\section{Results and Discussion}

\section{Bulk Properties}

The initial bulk density of silica in CG representation has been determined on the basis of the atomic positions in $\alpha$-quartz at zero temperature. During equilibration at $100 \mathrm{~K}$ we have observed a $14 \%$ reduction in the bulk density relative to the initial state. In particular, the density of CG silica $\left(2.268 \mathrm{~g} / \mathrm{cm}^{3}\right)$ differs from the density of natural quartz $\left[2.649 \mathrm{~g} / \mathrm{cm}^{3}(45)\right]$. The bulk density for PS has been obtained as $1.158 \mathrm{~g} / \mathrm{cm}^{3}$ and compares favorably with the findings of a previous CG study (32) in which a density of $1.020 \pm 0.00043 \mathrm{~g} / \mathrm{cm}^{3}$ at $300 K$ was reported (Tables 2 and 3).

Elastic moduli of the bulk PS are reported in Appendix 2. The isotropic constants obtained by the least-square fit read: Young's modulus $E_{p}=725 \mathrm{MPa}$, Poisson's ratio $v_{p}=0.336$, bulk modulus $K_{p}=736 \mathrm{MPa}$, Lamé's first modulus $\lambda_{p}=555 \mathrm{MPa}$, shear modulus $\mu_{p}=271 \mathrm{MPa}$. The specified values are in a good

Table 2. A summary of the off-diagonal $\left(\mathrm{C}^{12}, \mathrm{C}^{13}, \mathrm{C}^{23}\right)$ elastic moduli, as well as the Young's moduli in each direction $\left(\mathrm{E}^{1}, \mathrm{E}^{2}, \mathrm{E}^{3}\right)$ and the bulk (K) modulus (in MPa) for the NCs and bulk PS (first row) as obtained from MD simulations.

\begin{tabular}{|c|c|c|c|c|c|c|c|}
\hline $\mathrm{R}_{0}$ & $\mathrm{C}^{11}$ & $\mathrm{C}^{13}$ & $\mathrm{C}^{23}$ & K & $E^{1}$ & $E^{2}$ & $E^{3}$ \\
\hline- & $550 \pm 27$ & $556 \pm 28$ & $564 \pm 29$ & $727 \pm 9$ & $724 \pm 46$ & $712 \pm 39$ & $703 \pm 42$ \\
\hline 23.59 & $488 \pm 98$ & $485 \pm 95$ & $476 \pm 98$ & $650 \pm 34$ & $720 \pm 140$ & $658 \pm 150$ & $694 \pm 147$ \\
\hline 26.79 & $487 \pm 87$ & $516 \pm 85$ & $497 \pm 81$ & $649 \pm 26$ & $663 \pm 120$ & $708 \pm 123$ & $679 \pm 131$ \\
\hline 29.57 & $508 \pm 73$ & $541 \pm 75$ & $514 \pm 72$ & $655 \pm 23$ & $652 \pm 101$ & $656 \pm 99$ & $680 \pm 103$ \\
\hline 32.25 & $511 \pm 67$ & $486 \pm 65$ & $486 \pm 67$ & $642 \pm 22$ & $668 \pm 95$ & $717 \pm 93$ & $673 \pm 89$ \\
\hline 36.56 & $510 \pm 53$ & $493 \pm 50$ & $505 \pm 51$ & $658 \pm 16$ & $685 \pm 67$ & $665 \pm 72$ & $672 \pm 70$ \\
\hline 42.99 & $519 \pm 44$ & $513 \pm 44$ & $534 \pm 43$ & $665 \pm 13$ & $687 \pm 58$ & $694 \pm 60$ & $655 \pm 56$ \\
\hline
\end{tabular}


Table 3. A summary of the diagonal $\left(\mathrm{C}^{11}, \mathrm{C}^{22}, \mathrm{C}^{33}, \mathrm{C}^{44}, \mathrm{C}^{55}, \mathrm{C}^{66}\right.$ ) elastic moduli (in MPa) for the NCs and bulk PS (first row) as obtained from MD simulations.

\begin{tabular}{|c|c|c|c|c|c|c|}
\hline $\mathrm{R}_{0}$ & $\mathrm{C}^{11}$ & $\mathrm{C}^{22}$ & $\mathrm{C}^{33}$ & $\mathrm{C}^{44}$ & $\mathrm{C}^{55}$ & $C^{66}$ \\
\hline- & $1102 \pm 43$ & $1098 \pm 41$ & $1088 \pm 40$ & $281 \pm 28$ & $259 \pm 28$ & $283 \pm 26$ \\
\hline 23.59 & $990 \pm 146$ & $970 \pm 136$ & $1031 \pm 134$ & $246 \pm 97$ & $219 \pm 96$ & $253 \pm 87$ \\
\hline 26.79 & $1039 \pm 121$ & $1057 \pm 123$ & $993 \pm 129$ & $248 \pm 75$ & $268 \pm 85$ & $248 \pm 78$ \\
\hline 29.57 & $1002 \pm 102$ & $983 \pm 107$ & $1002 \pm 108$ & $250 \pm 61$ & $242 \pm 63$ & $238 \pm 64$ \\
\hline 32.25 & $989 \pm 103$ & $963 \pm 97$ & $979 \pm 80$ & $247 \pm 57$ & $261 \pm 54$ & $225 \pm 51$ \\
\hline 36.56 & $991 \pm 72$ & $986 \pm 79$ & $1030 \pm 77$ & $251 \pm 48$ & $260 \pm 50$ & $256 \pm 47$ \\
\hline 42.99 & $1019 \pm 64$ & $1017 \pm 63$ & $1015 \pm 61$ & $266 \pm 36$ & $261 \pm 38$ & $265 \pm 43$ \\
\hline
\end{tabular}

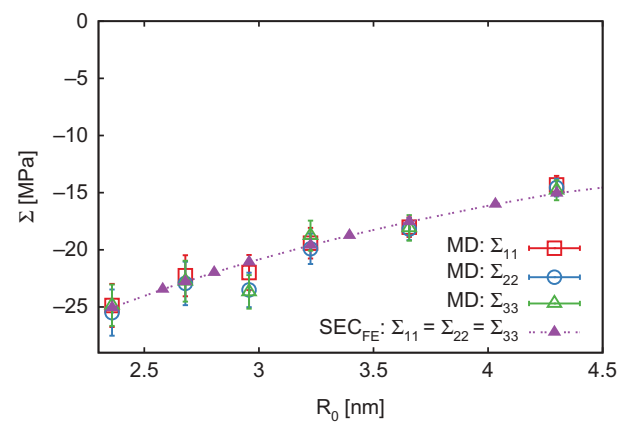

(a) Relaxation under interface tension

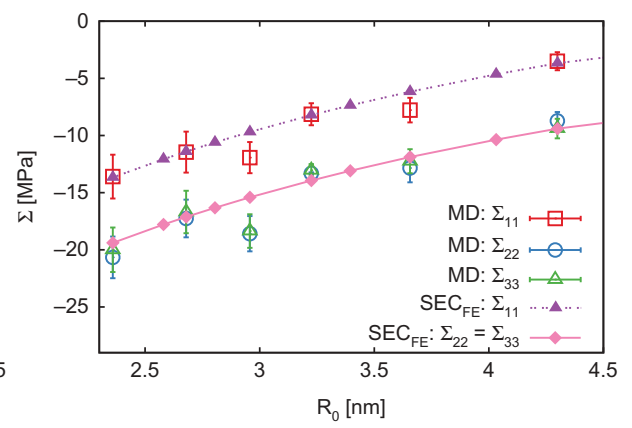

(b) Uniaxial deformation of $1 \%$

Fig. 4. The observed size effect for the nanocomposite system in terms of macroscopic Cauchy stress. (a) corresponds to the configuration that is common to both SEC and MD (see Fig. 1b). Negative pressure indicates negative interface tension; (b) corresponds to the 1\% uniaxial deformation applied to the state depicted in (a).

agreement with Young's and bulk moduli obtained from additional simulations (Table 2). Our results also agrees with the Young's modulus of approximately $715 \mathrm{MPa}$ reported in Rahimi et al. [3, see Figure 8].

Elastic moduli of the bulk $\mathrm{CG} \mathrm{SiO}_{2}$ (in Voight notation) are obtained as ${ }^{4}$ (in GPa) $\mathrm{C}_{\mathrm{np}}^{11}=195.75, \mathrm{C}_{\mathrm{np}}^{12}=71.47, \mathrm{C}_{\mathrm{np}}^{13}=84.20$, $\mathrm{C}_{\mathrm{np}}^{16}=-17.93, \mathrm{C}_{\mathrm{np}}^{33}=208.21, \mathrm{C}_{\mathrm{np}}^{44}=75.75$. Those values are different from the experimental elastic moduli of $\alpha$-quartz (in GPa) $\mathrm{C}^{11}=86.8, \mathrm{C}^{12}=7.04, \mathrm{C}^{13}=11.91, \mathrm{C}^{14}=-18.04$, $\mathrm{C}^{33}=105.75, \mathrm{C}^{44}=58.20(45)$. For the comparison between $\mathrm{MD}$ and SEC results, however, this issue is not relevant as long as bulk properties used in CM simulations are consistent with those obtained from the MD simulations.

To allow a better comparison of FE-SEC results to analytical approaches that consider isotropic behavior only, the NP is described as an isotropic neo-Hookean hyperelastic material with $\mu_{\mathrm{np}}=63.72 \mathrm{GPa}$ and $\lambda_{\mathrm{np}}=76.21 \mathrm{GPa}$. These values are obtained by the least-square fit of the anisotropic elastic moduli.

\section{Relaxation Under Interface Tension}

We focus first on the stress state developed in two scenarios, namely, the configuration relaxed due to interface tension (Fig. 1b), and its uniaxial deformation of $1 \%$. The results for

${ }^{4}$ Note the absence of error ranges as the results are obtained at zero temperature.
MD and SEC approaches are compared in Fig. 4. As is evident from the MD results, the macroscopic behavior of the system during relaxation under interface tension is close to isotropic even though the silica NP has an anisotropic elastic tensor. In particular, principal components of the resulting macroscopic stresses developing due to interface tension are nearly the same. Similarly, the transverse components of stress are almost equal upon application of uniaxial deformation. This justifies the use of neo-Hookean models for both interface and NP.

For the SEC model, the three unknown interface parameters $\hat{\mu} / \mu_{\mathrm{np}}=1.1453 \mathrm{~nm}, \hat{\lambda} / \lambda_{\mathrm{np}}=3.8306 \mathrm{~nm}$, and $\widehat{\gamma} / \mu_{\mathrm{np}}=-0.5221 \mathrm{~nm}$ are obtained by the least square fit of the MD results. To that end, the Nelder-Mead derivative-free simplex method (46) provided by Octave (47) was utilized. To minimize computational costs, only the three highest radii are considered during optimization and the ratio $\widehat{\mu} / \bar{\lambda}$ was kept fixed. The SEC theory provides a satisfactory fit of MD data (Fig. 4).

\section{Macroscopic Elastic Moduli}

The variation of the normalized components of the stiffness matrix $\mathrm{C}$ as well as of the bulk and Young's moduli as a function of the NP radius is shown in Fig. 5 and Fig. 6. Normalization was performed using the corresponding bulk PS moduli. The exact values and their standard deviations are tabulated within Tables 2 and 3. 


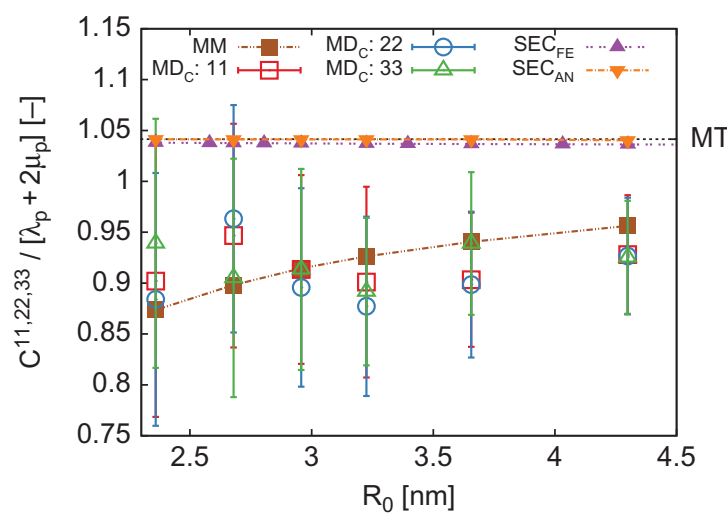

(a) Unidirectional modulus

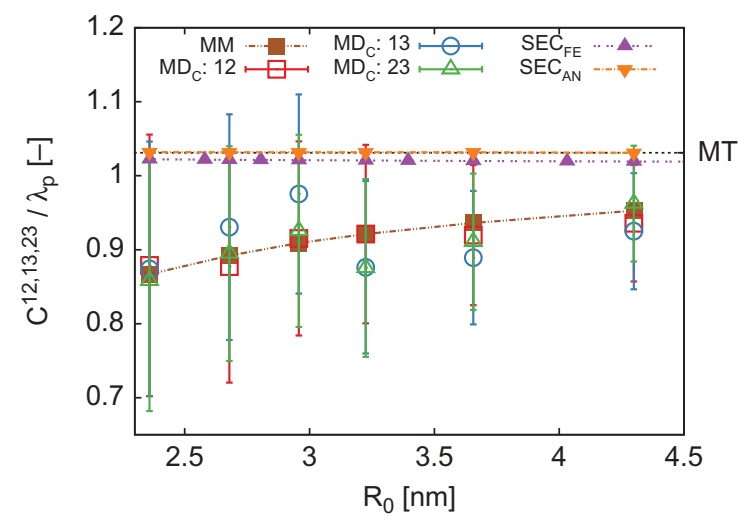

(b) Lamé modulus

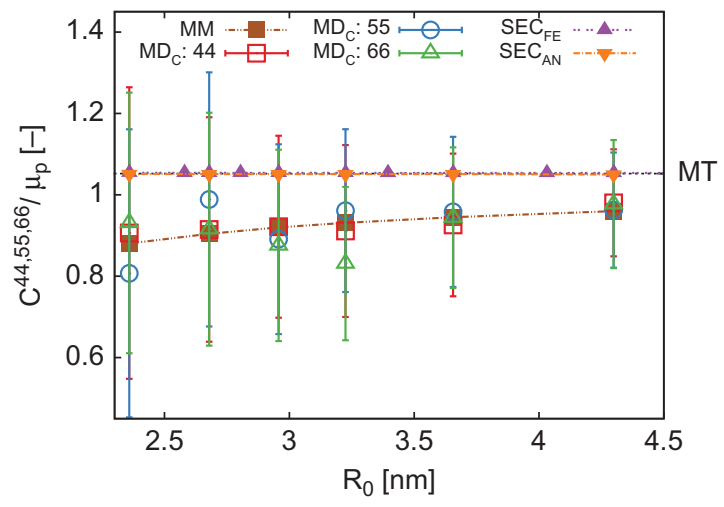

(c) Shear modulus

Fig. 5. The observed size effect for the NC systems in terms of computed elastic moduli. All values are plotted normalized with respect to the corresponding properties of bulk PS. For the MD simulations, (a), (b), and (c) show a complete set of (anisotropic) NCs elastic moduli. These are obtained from uniaxial deformation and simple shear and denoted by $\mathrm{MD}_{\mathrm{c}}$. $\mathrm{SEC}_{\mathrm{FE}}$ and $\mathrm{SEC}_{\mathrm{AN}}$ denote $\mathrm{FE}$ and analytical solutions of SEC theory, whereas MM indicate results obtained by the micro-mechanical 3 phase approach. Finally, MT denotes values obtained by Mori-Tanaka homogenization.

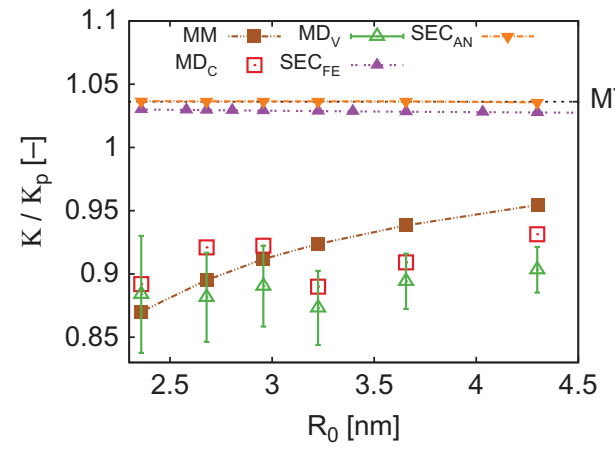

(a) Bulk modulus

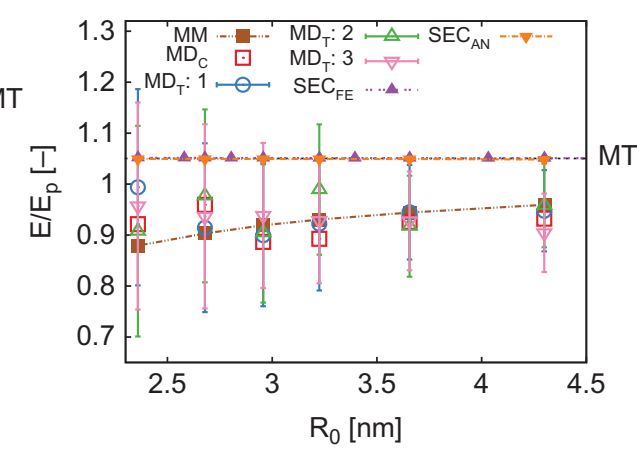

(b) Young modulus

Fig. 6. The observed size effect for the NC systems in terms of computed elastic moduli. All values are plotted normalized with respect to the corresponding properties of bulk PS. Young's and bulk moduli as evaluated from uniaxial tension and volumetric deformation using MD simulations are denoted by $\mathrm{MD}_{\mathrm{T}}$ and $\mathrm{MD}_{\mathrm{V}}$, respectively. Bulk and Young's moduli resulting from a least-square fit of general elastic mouduli $\mathrm{C}^{\mathrm{ij}}$ are denoted by $\mathrm{MD}_{\mathrm{c}}$. $\mathrm{SEC}_{\mathrm{FE}}$ and $\mathrm{SEC}_{\mathrm{AN}}$ denote $\mathrm{FE}$ and analytical solutions of SEC theory, whereas MM indicate results obtained by the micro-mechanical 3 phase approach. Finally, MT denotes values obtained by Mori-Tanaka homogenization.

With reference to Fig. 5 and Tables 2 and 3, for a given radius, we observe that the differences among $\mathrm{C}^{11}, \mathrm{C}^{22}, \mathrm{C}^{33}$; and $\mathrm{C}^{44}$, $\mathrm{C}^{55}, \mathrm{C}^{66}$; and $\mathrm{C}^{12}, \mathrm{C}^{13}, \mathrm{C}^{23}$, as determined by $M D$ simulations, are rather small. In consideration of the statistical uncertainties, the studied NC materials can be classified as isotropic.
As it is evident from Fig. 5, the overall elastic moduli are lower than those of the bulk PS. From this result, it is hypothesized that improved NC properties can only be observed by utilizing surface treated NPs. One possible way to achieve this would be to graft polymer chains onto the NP surface $(48,49)$. 
With regard to continuum approaches, the change of elastic moduli for different radii as predicted using $S E C_{A N}$ and $S E C_{\mathrm{FEM}}$ are negligible at this scale. The micro-mechanical three phase model results in a satisfactory fit to the observed elastic moduli (Fig. 5 and Fig. 6). The interphase elastic moduli are fitted in a least-square sense using Octave (47) and resulted in $\mu_{\text {int }} / \mu_{\mathrm{p}}=0.244$ and $\lambda_{\text {int }} / \lambda_{\mathrm{p}}=0.325$. Although the size dependence is clearly pronounced, this approach does not show a perfect correlation with the $M D$ data either. In the limit, values predicted by $S E C_{A N}, M M$, and $M D$ appear to converge to those predicted by the $M T$ approach.

To gain further insights into the elastic moduli size dependence, local structural and dynamical properties of the interphase have to be studied.

\section{Discussion on the Application of the SEC Theory}

The lack of correlation between elastic moduli as predicted by SEC and MD suggests the importance of the PS-NP interphase, which is in this instance assumed to be of zero thickness in SEC theory. Indeed, the estimated NC's interphase thickness of $2.0 \mathrm{~nm}$ is not negligible when compared to the NP radius and the RVE size that are in the order of $10 \mathrm{~nm}$ (Table 1).

As can be observed from Fig. 4, the imposed stresses are of a compressive nature. In terms of the SEC theory, this implies a negative interface energy. Negative values for the surface elastic constants are admissible. From a mathematical point of view, the admissible range for the surface elastic constants could be calculated from an instability analysis similar to that in the bulk. These types of local analyses verify that wave-like instabilities occur beyond the point where the surface elasticity moduli loose pointwise stability (50). It should be emphasized that, critically, the admissible range for the surface material properties in the local SEC theory does not necessarily include the values as estimated from inherently non-local atomistic models. Note that the latter are of higher accuracy from a physical point of view. In particular, surface material parameters can be negative, whereas the analogous bulk material parameters are strictly positive. This can be justified by the fact that the surface cannot exist independent of the bulk and the overall response of the bulk together with the surface needs to be positive definite (44).

It is known that negative values of the interface elastic constants may result in numerical problems (38). Ultimately this leads to the restriction of a minimum mesh size, below which calculations are unstable. This length scale required for stability has no physical justification. For the current studies a rather coarse mesh had to be considered in order to perform SEC simulations with the resulting negative interface tension. This raises the question as to whether the FE-SEC results are meaningful. However, the analytical SEC homogenization method does not suffer from such restrictions. Nonetheless it was not possible to attain a satisfactory correlation between its results and MD. To that end, a least square fit of bulk and shear moduli was attempted using gradient and simplex minimization routines in Octave (47). Note, the adopted analytical model does not consider interface tension, but only bulk and shear surface moduli, both of which are positive in the current study. This simplification could be a contributing factor to the lack of correlation with the MD results. Additionally, the FE-SEC calculations with zero surface tension and parameters specified in the aforementioned were performed. The results are only marginally different from the analytical SEC homogenization approach and do not exhibit a pronounced size effect either. Thus, we can conclude that the local SEC theory does not provide satisfactory results as compared to the non-local MD simulations.

The failure of the SEC approach to capture the size effect in this case is due to the large difference in the mechanical properties between PS and NP. In porous media it has been shown (51) that, although the surface properties can provide a size effect with respect to the pores diameter, such an influence has an upper limit. For mechanically strong surfaces a void behaves almost as a rigid particle, a result that has been confirmed both by the $\mathrm{SEC}_{\mathrm{FE}}$ and the $\operatorname{SEC}_{\mathrm{AN}}$ (51). When considering a particulate composite, a particle such as the NP is already very strong compared to the matrix (PS), and thus the surface cannot attribute further to the NC's response.

\section{Conclusions}

In the present work we have analyzed the influence of nanoparticle size on the mechanical properties of systems composed of a bare spherical silica nanoparticle and a glassy polystyrene matrix. We employ coarse grained molecular dynamics simulations and surface enhanced continuum theory, as well as the micro-mechanical model.

The anisotropic elastic moduli are obtained by applying smallstrain deformation modes to the glassy structures. From the computed data, we conclude that the nanocomposites exhibit near isotropic mechanical behavior. Equivalent isotropic constants are estimated using the least-square fit of the obtained stiffness matrix. The elastic moduli of the nanocomposites are lower than those of the pure polymer matrix. In the limit, the observed elastic moduli appear to converge from below toward those predicted by a macroscopic Mori-Tanaka model.

An attempt was made to fit the molecular dynamics results using the surface enhanced continuum approach. It is argued that in order to study size-effects consistently, one needs to consider nanocomposites of the same mass fraction. Assuming a neo-Hookean behavior, bulk elastic moduli of nanoparticle and polystyrene matrix were estimated from the molecular dynamics simulations. The development of negative stresses in the molecular dynamics results indicates that the nanocomposites studied here have a negative interface energy. Interface parameters for the surface constitutive model were obtained by the least square fit of molecular dynamics results using the Nelder-Mead simplex method (46). A satisfactory fit of macroscopic stresses developing during relaxation due to the interface tension, as well as during uniaxial deformation, were obtained for all radii. However, it was not possible to quantify with confidence the dependence of the nanocomposites' elastic moduli on nanoparticle's radius. Both finite elements and analytical solutions of surface enhanced continuum theory produced results conflicting with those obtained from the molecular dynamics simulations. The observed deviations for the systems under consideration could be attributed to the large difference in the mechanical properties of the polymer matrix and the nanoparticle. In addition, a micro-mechanical analytical model was examined. Although 
its results are somewhat better, both quantitative and qualitative discrepancy to the previously mentioned results is evident.

Finally, note that a higher order non-local continuum theory would also lead to a size-dependent solution. It was shown using detailed atomistic simulations that characteristic length scales in strain gradient elasticity are not negligible for polymeric materials $(52,53)$. A second-order interface description would require a third-order bulk theory (54). In such a case both the continuum and discrete models are non-local; thus, a better agreement is expected. Only the combined (surface and bulk) size-effect could be observed from MD using the aforementioned methodology.

\section{Acknowledgement}

The first author is grateful to Ali Javili and Andrew McBride for numerous fruitful discussions on issues related to surface elasticity and its numerical implementation using the FE approach, as well as to Jean-Paul Pelteret for critical comments during the manuscript preparation.

\section{Funding}

The financial support of the German Science Foundation (Deutsche Forschungs-Gemeinschaft, DFG) within the priority program 1369 "Polymer-solid contacts: Interphases and interfaces" is gratefully acknowledged. The support of this work by the ERC Advanced Grant MOCOPOLY is also gratefully acknowledged.

\section{References}

1. Ghanbari, A., Ndoro, T. V. M., Leroy, F., Rahimi, M., Böhm, M. C., and Müller-Plathe, F. (2012). Interphase structure in silicapolystyrene nanocomposites: A coarse-grained molecular dynamics study. Macromolecules, 45(1):572-584.

2. Ndoro, T. V. M., Voyiatzis, E., Ghanbari, A., Theodorou, D. N., Böhm, M. C., and Müller-Plathe, F. (2011) Interface of grafted and ungrafted silica nanoparticles with a polystyrene matrix: Atomistic molecular dynamics simulations. Macro-molecules, 44(7):2316-2327.

3. Rahimi, M., Iriarte-Carretero, I., Ghanbari, A., Böhm, M. C., and Müller-Plathe, F. (2012) Mechanical behavior and interphase structure in a silica-polystyrene nanocomposite under uniaxial deformation. Nanotechnology, 23(30):305702.

4. Zhao, J., Nagao, S., Odegard, G. M., Zhang, Z., Kristiansen, H., and He, J. (2013) Size-dependent mechanical behavior of nanoscale polymer particles through coarsegrained molecular dynamics simulation. Nanoscale Res. Lett., 8(1):1-26.

5. Pfaller, S., Rahimi, M., Possart, G., Steinmann, P., Müller-Plathe, F., and Böhm, M. C. (2013) An Arlequin-based method to couple molecular dynamics and finite element simulations of amorphous polymers and nanocomposites. Computer Method. Appl. Mechan. Eng., 260:109-129.

6. Yang, S., Choi, J., and Cho, M. (2012) Elastic stiffness and filler size effect of covalently grafted nanosilica polyimide composites: Molecular dynamics study. ACS Appl. Mater. Interface., 4(9):4792-4799.

7. Adnan, A., Sun, C. T., and Mahfuz, H. (2007) A molecular dynamics simulation study to investigate the effect of filler size on elastic properties of polymer nanocomposites. Composite. Sci. Technol., 67(3-4):348-356.

8. Cho, J., and Sun, C. T. (2007) A molecular dynamics simulation study of inclusion size effect on polymeric nanocomposites. Comput. Mater. Sci., 41(1):54-62.
9. Cho, J., Joshi, M. S., and Sun, C. T. (2006) Effect of inclusion size on mechanical properties of polymeric composites with micro and nano particles. Compos. Sci. Technol., 66(13):1941-1952.

10. Chisholm, N., Mahfuz, H., Rangari, V. K., Ashfaq, A., and Jeelani, S. (2005) Fabrication and mechanical characterization of carbon/SiCepoxy nanocomposites. Compos. Struct., 67(1):115-124.

11. Tsai, J. L., and Tzeng, S. H. (2008) Characterizing mechanical properties of particulate nanocomposites using micromechanical approach. $J$. Compos. Mater., 42(22):2345-2361.

12. Kontou, E., and Anthoulis, G. (2007) The effect of silica nanoparticles on the thermo-mechanical properties of polystyrene. J. Appl. Polymer Sci., 105(4):1723-1731.

13. Brown, D., Mélé, P., Marceau, S., and Albérola, N. D. (2003) A molecular dynamics study of a model nanoparticle embedded in a polymer matrix. Macromolecules, 36(4):1395-1406.

14. Odegard, G. M., Clancy, T. C., and Gates, T. S. (2005) Modeling of the mechanical properties of nanoparticle/polymer composites. Polymer, 46(2):553-562.

15. Li, X. H., Tjong, S. C., Meng, Y. Z., and Zhu, Q. (2003) Fabrication and properties of polypropylene carbonate)/calcium carbonate composites. J. Polymer Sci., Part B, 41(15):1806-1813.

16. Boutaleb, S., Zaïri, F., Mesbah, A., Naï-Abdelaziz, M., Gloaguen, J. M., Boukharouba, T., and Lefebvre, J. M. (2009) International Journal of Solids and Structures. Int. J. Solid. Struct., 46(7-8):1716-1726.

17. Marcadon, V., Brown, D., Hervé, E., Mélé, P., Albérola, N. D., and Zaoui, A. (2013) Confrontation between Molecular Dynamics and micromechanical approaches to investigate particle size effects on the mechanical behaviour of polymer nanocomposites. Comput. Mater. Sci., 79:495-505.

18. Brown, D., Marcadon, V., Mélé, P., and Albérola, N. D. (2008) Effect of filler particle size on the properties of model nanocomposites. Macromolecules, 41(4):1499-1511.

19. Gurtin, M. E., and Ian Murdoch, A. (1975) A continuum theory of elastic material surfaces. Arch. Rat. Mechan. Anal., 57(4):291-323.

20. Javili, A., and Steinmann, P. (2009) A finite element framework for continua with boundary energies. Part I: The two-dimensional case. Computer Method. Appl. Mechan. Eng., 198(27-29):2198-2208.

21. Davydov, D., Javili, A., and Steinmann, P. (2013) On molecular statics and surface-enhanced continuum modeling of nano-structures. Comput. Mater. Sci., 69:510-519.

22. Davydov, D., Javili, A., Steinmann, P., and McBride, A. (2013) A comparison of atomistic and surface enhanced continuum approaches at finite temperature, pp:43-57 Surface Effects in Solid Mechanics; Springer Verlag: Berlin Heidelberg, Germany.

23. Davydov, D., and Steinmann, P. (2014) Reviewing the roots of continuum formulations in molecular systems. Part I: Particle dynamics, statistical physics, mass and linear momentum balance equations. Math. Mechan. Solid., 19: 411-433.

24. Davydov, D., and Steinmann, P. (2013) Reviewing the roots of continuum formulations in molecular systems. Part II: Energy and angular momentum balance equation. Math. Mechan. Solid., 1-16.

25. Davydov, D., and Steinmann, P. (2013) Reviewing the roots of continuum formulations in molecular systems. Part III: Stresses, couple stresses, heat fluxes. Math. Mechan. Solid., 1-17.

26. Sharma, P., and Ganti, S. (2004) Size-dependent eshelby's tensor for embedded nanoinclusions incorporating surface/interface energies. $J$ Appl. Mechan., 71(5):663-671.

27. Duan, H. L., Yi, X., Huang, Z. P., and Wang, J. (2007) A unified scheme for prediction of effective moduli of multiphase composites with interface effects: Part II-Application and scaling laws. Mechan. Mater., 39(1):94-103.

28. Duan, H. L., Wang, J., Huang, Z. P., and Karihaloo, B. L. (2005) Size-dependent effective elastic constants of solids containing nanoinhomogeneities with interface stress. Journal of the Mechanics and Physics of Solids, 53(7):1574-1596.

29. Altenbach, H., Eremeyev, V. A., and Morozov, N. F. (2012) Surface viscoelasticity and effective properties of thin-walled structures at the nanoscale. Int. J. Eng. Sci., 59:83-89. 
30. Eremeyev, V. A., Altenbach, H., and Morozov, N. F. (2009) The influence of surface tension on the effective stiffness of nanosize plates. Doklady Phys., 54(2):98-100.

31. Chatzigeorgiou, G., Javili, A., and Steinmann, P. (2014) Surface electrostatics: Theory and computations. Proceed. Math. Phys. Eng. Sci. / The Royal Soc., 470(2164):20130628.

32. Rahimi, M., Karimi-Varzaneh, H. A., Böhm, M. C., Müller-Plathe, F., Pfaller, S., Possart, G., and Steinmann, P. (2011) Nonperiodic stochastic boundary conditions for molecular dynamics simulations of materials embedded into a continuum mechanics domain. J. Chem. Phys., 134(15): 154108.

33. Reith, D., Pütz, M., and Müller-Plathe, F. (2003) Deriving effective mesoscale potentials from atomistic simulations. J. Comp. Chem., 24(13):1624-1636.

34. Frenkel, D., and Smit, B. (2001) Understanding Molecular Simulation: From Algorithms to Applications. Academic Press: San Diego, California, USA.

35. Verlet, L. (1967) Computer "experiments" on classical fluids. I. Thermodynamical properties of Lennard-Jones molecules. Phys. Rev.

36. Plimpton, S. (1995) Fast parallel algorithms for short-range molecular dynamics. J. Comp. Phys., 117(1):1-19.

37. Theodorou, D. N., and Suter, U. W. (1986) Atomistic modeling of mechanical properties of polymeric glasses. Macromolecules, 19(1):139-154.

38. McBride, A., Javili, A., Steinmann, P., and Reddy, B. D. (2014) A finite element implementation of surface elasticity at finite strains using the deal.II library. Submitted.

39. Javili, A., McBride, A., Steinmann, P., and Reddy, B. D. (2014) A unified computational framework for bulk and surface elasticity theory: a curvilinear-coordinate-based finite element methodology. Comput. Mechan., 1-27.

40. Bangerth, W., Hartmann, R., and Kanschat, G. (2007) Deal.II-A general-purpose object-oriented finite element library. ACM Transact. Math. Software, 33(4):1-27.

41. Mori, T., and Tanaka, K. (1973) Average stress in matrix and average elastic energy of materials with misfitting inclusions. Acta Metall., 21(5):571-574.

42. Eshelby, J. D. (1957) The determination of the elastic field of an ellipsoidal inclusion, and related problems. Proceed. Royal Soc. A, 241(1226):376-396.

43. Nozières, P., and Wolf, D. E. (1988) Interfacial properties of elastically strained materials - I. Thermodynamics of a planar interface. Zeitschrift Phys. B, 70(3):399-407.

44. Shenoy, V. (2005) Atomistic calculations of elastic properties of metallic fcc crystal surfaces. Phys. Rev. B, 71(9):094104.

45. Lide, D. R. (2004) CRC Handbook of Chemistry and Physics, 85th Ed.; Taylor and Francis: Boca Raton, Florida, USA.
46. Nelder, J. A., and Mead, R. (1965) A simplex method for function minimization. Comp. J., 7(4):308-313.

47. Octave Community. (2014) GNU Octave 3.8.1. Technical Report.

48. Maillard, D., Kumar, S. K., Fragneaud, B., Kysar, J. W., Rungta, A., Benicewicz, B. C., Deng, H., Brinson, L. C., and Douglas, J. F. (2012) Mechanical properties of thin glassy polymer films filled with spherical polymer-grafted nanoparticles. Nano Lett., 12(8):3909-3914.

49. Kumar, S. K., Jouault, N., Benicewicz, B., and Neely, T. (2013) Nanocomposites with polymer grafted nanoparticles. Macromolecules, 46(9):3199-3214.

50. Javili, A., McBride, A., Steinmann, P., and Reddy, B. D. (2012) Relationships between the admissible range of surface material parameters and stability of linearly elastic bodies. Phil. Mag., 92(28-30):3540-3563

51. Javili, A., McBride, A., Mergheim, J., Steinmann, P., and Schmidt, U. (2013) Micro-to-macro transitions for continua with surface structure at the microscale. Int. J. Solid. Struct., 50(16-17):2561-2572.

52. Maranganti, R., and Sharma, P. (2007) A novel atomistic approach to determine strain-gradient elasticity constants: Tabulation and comparison for various metals, semiconductors, silica, polymers and the (Ir) relevance for nanotechnologies. J. Mechan. Phys. Solid., 55(9): $1823-1852$

53. Maranganti, R., and Sharma, P. (2007) Length scales at which classical elasticity breaks down for various materials. Phys. Rev. Lett., 98(19): 195504

54. Javili, A., dell'Isola, F., and Steinmann, P. (2013) Geometrically nonlinear highergradient elasticity with energetic boundaries. J. Mechan. Phys. Solid., 61(12):2381-2401.

\section{Appendix 1. Notation and Definitions}

Direct notation is adopted throughout. The scalar product of two vectors $\boldsymbol{a}$ and $\boldsymbol{b}$ is denoted $\boldsymbol{a} \cdot \boldsymbol{b}=[\boldsymbol{a}]_{i}[\boldsymbol{b}]_{i}$. The scalar product of two second-order tensors $\boldsymbol{A}$ and $\boldsymbol{B}$ is denoted $\boldsymbol{A}: \boldsymbol{B}=$ $[\boldsymbol{A}]_{i j}[\boldsymbol{B}]_{i j}$. The composition of two second-order tensors $\boldsymbol{A}$ and $\boldsymbol{B}$, denoted $\boldsymbol{A} \cdot \boldsymbol{B}$, is a second-order tensor with components $[\boldsymbol{A} \cdot \boldsymbol{B}]_{i j}=[\boldsymbol{A}]_{i m}[\boldsymbol{B}]_{m j}$. The action of a second-order tensor $\boldsymbol{A}$ on a vector $\boldsymbol{a}$ is provided by $[\boldsymbol{A} \cdot \boldsymbol{a}]_{i}=[\boldsymbol{A}]_{i j}[\boldsymbol{a}]_{j}$. The gradient of a quantity $\{\bullet\}$ with respect to the material configuration is denoted as $\{\bullet\} \otimes \nabla_{\boldsymbol{X}}$. Let $\boldsymbol{I}$ denote the identity tensors in the material configuration. Divergence operator with respect to the material configuration is denoted as Div and is defined by $\operatorname{Div}\{\bullet\}=$ $\{\bullet\} \cdot \nabla_{\boldsymbol{X}}$. All quantities attributed to the surface/interface are denoted with $\{\bullet\}$. 